\title{
Materialidad de los cuerpos sin mundo: la función ética del hambre del otro'
}

\author{
Dra. Claudia Gutiérrez Olivares²
}

Recibido: 25 de febrero de 2019 . Aprobado: 24 de abril de 2019

\begin{abstract}
Resumen
El siguiente texto explora la noción de "alteridad" como una categoría asentada en una lógica de la exclusión. La alteridad del excluido encuentra en su génesis la huella de una expulsión del mundo, en la que la categoría del "hambriento" parece disponer de una autoridad particular, y que este texto intenta subrayar. La alteridad, en este sentido, es sinónimo de destierro, de apartamiento del mundo $y$, si se nos permite inventar una palabra, de des-mundo. Bajo este respecto, una filosofía de la materialidad de los cuerpos sin mundo puede ser la indicación del paradigma de la injusticia, en la cual la filosofía ética de Lévinas es un notable intento de respuesta.
\end{abstract}

Palabras clave: alteridad - ética - mundo - hambre - vulnerabilidad

\section{Materiality of world-less bodies: the ethical function of other's hunger}

\begin{abstract}
The following text explores the notion of "otherness" as a category grounded in a logic of exclusion. The otherness of the excluded is, in its origin, the mark of expulsion from the world, where the category of "hungry" appears to have a particular authority that this text intends to highlight. Otherness, in this sense, is a synonym of exile, separation from the world and, if we may invent a word, world-lessness. In this regard, the philosophy of the materiality of world-less bodies may be the indication
\end{abstract}

1 Este texto reelabora parte de la ponencia presentada en el Simposio "Lévinas y la dimensión política de la alteridad", en el IV Congreso Internacional de la Sociedad Filosófica del Uruguay (SFU), 6 a 9 de junio de 2018, Montevideo, Uruguay.

2 Chilena, Licenciada y Magíster en Filosofía por la Universidad de Chile, Chile. Doctora en Filosofía por la Universidad Paris VIII, Francia. Académica del Departamento de Filosofía, Universidad de Chile. Contacto: clgutierrez@uchile.cl

ORCID: http://orcid.org/0000-0002-4118-9669 
of a paradigm of injustice, in which the ethical philosophy of Levinas is a notable attempt to respond.

Key words: otherness - ethics - world - hunger - vulnerability

\title{
Materialidade dos corpos sem mundo: a função ética da fome do outro
}

\begin{abstract}
Resumo
O seguinte texto explora a noção de "alteridade" como uma categoria baseada em uma lógica da exclusão. A alteridade do excluído encontra em sua gênese a marca de uma expulsão do mundo, na qual a categoria do "faminto" parece dispor de uma autoridade particular, e que este texto tenta sublinhar. A alteridade, nesse sentido, é sinónimo de desterro, de afastamento do mundo e, se nos é permitido criar uma palavra, de desmundo. Sob esta visão, uma filosofia da materialidade dos corpos sem mundo pode ser a indicação do paradigma da injustiça, na qual a filosofia ética de Lévinas é uma notável tentativa de resposta.
\end{abstract}

Palavras-chave: alteridade - ética - mundo - fome - vulnerabilidade

\section{Introducción}

Nada nuevo diremos al afirmar que la noción de "alteridad" ordena el sentido general de la obra de Lévinas, de esta alteridad del otro que Lévinas define incansablemente a lo largo de su obra, en términos de radical o absoluta, intentado delimitar bajo estos términos la orientación ética que la noción de "alteridad" dispone en la obra del filósofo. En cuanto radical, en su comparecencia, la alteridad del otro no puede sino molestar, perturbar, incomodar, dejando entrever la hebra de un argumento que, en su desarrollo, puede resultar obstinado, sino acaso, insufrible. ¿De dónde viene esta insistencia, esta obstinación por el otro? ¿Por qué no basta con situar al otro en un discurso de la diferencia y desplegar allí una filosofía del reconocimiento y de la acogida, así sin más? ¿Por qué Lévinas insiste en llevarnos a esa filosofía incómoda, a esa metafísica del otro, según su expresión, en la que el otro viene como a traumatizar y a dislocar las buenas intenciones del sentido común y de la filosofía profesional? 
Y es que a menudo olvidamos que el encuentro con el otro, tiene la forma de una "fisión" (Lévinas 1999 277), según la expresión de nuestro autor. Es decir, en la experiencia del encuentro con el otro se produce una conmoción, o incluso, y siguiendo aquí el sentido del vocablo "fisión" (fission en francés), se recoge la idea de una desintegración, como cuando se habla de la fisión nuclear, en física, o de la fisión celular, en biología. En todos los casos, a lo que se alude es a una idea de "rompimiento" o a una escisión. Aplicado al encuentro interhumano, la fisión apunta a la conmoción que sufre el "sí mismo" (Ibíd.) en la tensión de un vínculo direccionado por la responsabilidad para con el otro. Esta idea de responsabilidad por el otro supone así un verdadero cambio de paradigma en la comprensión de la constitución del sujeto responsable. La subjetividad responsable invita a pensar, dirá Lévinas, un proceso de "desnucleamiento de mi substancialidad" (Ibíd.), algo así como un desplazamiento irremediable de las capas tectónicas que soportan la subjetividad del sujeto, y hacer de ese desplazamiento la condición de posibilidad de esta responsabilidad ética por el otro. ¿Qué significa esta responsabilidad? ¿Quién es el otro que pone a prueba mi substancialidad subjetiva?

Como decíamos, mucho se ha escrito sobre la alteridad en sentido levinasiano, subrayando la dimensión superabundante de sentido que sostiene el aparecer del otro que, en cuanto tal, escapa a la lógica del fenómeno que reduce todo aparecer al ser, y deja al otro en los márgenes de un pensamiento de corte trascendental. Si el otro es enigma y no fenómeno (Lévinas 2001), la alteridad del otro funciona como una categoría que no se reabsorbe en significaciones estancas y rígidas, y por lo mismo se abre paso aquí, quizás, una verdadera gramática de la perturbación asociada a la comparecencia misma del otro. Si el otro es enigma, lejos de ser la indicación de un misterio impasible, tan lejano que solo se dejaría contemplar a la distancia, más bien la alteridad del otro tiene la forma de una "carga aplastante" (Lévinas 1999 271).

Para intentar dar contenido a este traumatismo de la alteridad, conviene volvernos hacia uno de los horizontes comprensivos que 
sostiene el aparecer del otro, e intentar precisar algo así como las condiciones de aparecer de esta alteridad abrumadora del otro. Se trata de una suerte de paradoja. Lo que perturba, la alteridad, es al mismo tiempo la figuración de una precariedad. Es una paradoja no solo lógica sino además ética, según la cual la extrema fragilidad y vulnerabilidad del otro constituye, en el mismo gesto significativo, la condición de la grandeza ética del otro, esto es lo que Lévinas llama su "altura" (Lévinas 1990 237) y que, en cuanto tal, introduce el germen de la perturbación o la conmoción ética de la alteridad. Bajo este respecto, quizás convenga detenerse en el vocabulario que utiliza Lévinas para significar esta paradoja de lo menos que puede más, de la fragilidad que mandata con autoridad. La presencia del rostro abre la escena de lo que Lévinas nombra la "indigencia" (Id. 234) de lo humano, que no es una categoría abstracta, sino que cristaliza en categorías que no podemos pasar a la ligera. En efecto, se trata de la indigencia del "pobre y del extranjero" (Ibíd.), que en su trabajo profundo habría que pensarlas como categorías del "exilio" ( $(\mathrm{bi} d$.), tributarias de una condición humana definida en términos de "apátrida" (Lévinas 1975 54). La interrogante que se nos abre aquí, y que queremos indagar en este texto, es que estas categorías son, en su conjunto, categorías ontológicas de la exclusión. De ahí su fuerza patética que no puede sino conmover. La alteridad del excluido, infinitamente diferente a la alteridad del amado, del amigo, de alguien añorado, nos parece ser un principio orientador, en el que se sustenta convenientemente en último término, y originariamente, esta categoría de "alteridad". Si la alteridad nos conmueve, nos perturba, es porque ella actualiza en el otro la violencia de una exclusión o expulsión del mundo, de la segregación de una parte de la humanidad, perpetrada, según nos muestra la historia, por unos pocos. La alteridad en este sentido es sinónimo de destierro, de apartamiento y, si se nos permite inventar una palabra, de des-mundo. Es en esta dirección que el otro aparece cercado por la semántica del despojado, que es una indicación respecto del paradigma de la injusticia, en la cual la ética convoca irremediablemente una política. Veamos. 


\section{La metafísica del otro}

Una primera idea a observar es la idea de "metafísica", que recubre la reflexión sobre la alteridad en Lévinas. Ella describe esta filosofía del encuentro con el otro, refiere la experiencia de ese encuentro y postula que allí se ampara una orientación de sentido ético. Bajo este respecto, esta metafísica del otro dispone desde sus inicios de una profunda dimensión práctica. Por una parte, se anuncia aquí una praxis, toda vez que esta metafísica se funda en la experiencia concreta de la intersubjetividad, apegada a la experiencia mundana en la que circulan sujetos empíricos. El otro no es ni pensado ni ensoñado, más bien, y como postulara Sartre, el otro es, ante todo, el producto de un encuentro (1994), con todos los riesgos que conlleva encontrarse con una presencia viva aquí y ahora. En esta metafísica estamos lejos de una dimensión abstractiva del otro, abordable solo por una operación de cálculo trascendental, en la que el otro, pese a ser planteado como un "problema especial" (Husserl 2016 123) y de alguna manera salvaguardar así la alteridad propia del humano, distinta a la cosa, sucumbe al análisis intencional, que explica todos los intersticios de este fenómeno especial que resulta ser el otro. Abordar al otro desde una dimensión metafísica no significa abordar al otro como un problema, sino más bien, y según decíamos, como un enigma que pone en tensión la alteridad del rostro con el mundo, reconociendo en esta situación mundana el influjo de lo real como algo que pesa en la existencia y da contenido a la alteridad del otro, al tiempo que acorta o borra la distancia trascendental que separa las subjetividades. Pero esta metafísica no podría prolongarse así, sin más, en una filosofía del mundo, que hiciera de la premisa mundana la condición del despliegue de nuestra existencia. El tratamiento del mundo, bajo la pluma de Lévinas, es otro; podríamos decir que cuando Lévinas afirma el mundo, no plantea que éste sea un horizonte de significaciones posibles, en donde destacan y cobran sentido los fenómenos de la experiencia. A este respecto, la categoría de "rostro" en Lévinas (1990) es muy ilustrativa, pues el rostro delimita y pone en cuestión justamente una concepción mundana como afluente de significaciones, y propone una idea de mundo como "conjunto de 
nutrimentos", tal como lo enseñan las páginas de Totalidad e Infinito (1990). Aquí se opera un desplazamiento metodológico, que va desde una concepción del mundo, en términos de aquello que sustenta un despliegue teórico de nuestro ser en el mundo, a una dimensión práctica (Lévinas 1990). El mundo no es solo aquello que puedo conocer, sino aquello que en primer lugar puedo disfrutar (Ibíd.). En este sentido, la noción de rostro viene a componer un paisaje especulativo en el que la categoría de "mundo", como noción de contexto, se retira o se ausenta, pues el rostro, dirá Lévinas, "no es el des-cubrimiento de un mundo" (2001 270), esto es, su significación de rostro no le viene del mundo. Hemos elaborado en otra parte (Gutiérrez 2012) una comprensión respecto del lugar del mundo, en el orden del sentido de la filosofía de Lévinas.

En un sentido más específico, una intersubjetividad de nuevo cuño, como es la de Lévinas, es pensada en términos de una metafísica, toda vez que el otro se exhibe como rostro, cuya decantación conceptual acabada se entronca con la noción de "exterioridad" (1990). Así, en esta curiosa metafísica, se pone en obra lo que Lévinas indica en términos de: "el respeto de la exterioridad" (1990 33), siendo esta exterioridad o trascendencia la dimensión que soporta el aparecer del otro. Hay aquí una idea práctica, en la medida en que este respeto declarado no es una regla abstracta, no es el acatamiento u obediencia ciega de una ley sin contenido, a la manera de un precepto de bondad o intención de buen comportamiento hacia el otro. Esta metafísica no nos invita a ser buenos ni nobles con el otro. Se trata más bien de una actitud de observancia hacia el otro, una orientación de sentido centrada en lo que quisiera llamar aquí la "materialidad" del otro que viene a mi encuentro. Esta observancia, el hecho de tomar nota de esta materialidad, se traduce en acciones transformadoras del mundo del otro, y que implican, en un análisis acabado y posterior, una política, cuestión esta última que quedará solo anunciada en este texto.

¿En qué consiste esta materialidad del otro? Ella alude al carácter y especificidad del aparecer del otro, a la singularidad de esta manifestación, que me interpela y en la que se devela su consistencia ontológica. En esta materialidad del otro se registran las maneras cómo 
este aparecer -que es una vida, un cuerpo concreto, infinitamente trascendente al mío- se fundamenta en una estructura ontológica y existencial del orden de la precariedad. La materialidad del otro se manifiesta originariamente como una "indigencia", como una "miseria" (Lévinas 1990 234), una pobreza, según las palabras de Lévinas. En este sentido, la idea de "exterioridad" es una palabra clave que puede darnos noticia de esta materialidad, de esta concreción del otro, en la medida en que una filosofía de la intemperie parece insinuarse allí, algo así como la imposibilidad de un refugio o de una semántica de la protección. La materialidad del otro es pura exposición. Al detenernos en la categoría de exterioridad, subrayemos primeramente que la idea de exterioridad en Lévinas, y según nos enseña en su libro Totalidad e Infinito. Ensayo sobre la exterioridad, no remite a una semántica del espacio y del lugar. El ser exterior no es el ser espacial, que en cuanto tal es determinable o situable según un juego de horizontes y planos diferenciados, según la geometría de valles y planicies, como cuando predicamos la exterioridad de una casa o de un árbol, que es todo aquello que Husserl definía como variantes fenoménicas o "múltiples modos de aparición" (2016 55) del objeto percibido. La exterioridad del otro no juega en este plano de perspectivas y matices, propios a la escena fenomenológica en la que se sustenta la consistencia ontológica de las cosas. El otro es una exterioridad sin horizontes, sin escorzos, es decir incalculable, no sabemos dónde comienza ni dónde termina, el otro es, a juicio de Lévinas, una figuración de lo infinito (1990) o, lo que es lo mismo, es la forma de una exterioridad completamente abierta, que no se cierra sobre ningún horizonte, que de alguna manera está como saturada de su propia presencia, que no encuentra descanso ni remite a ninguna interioridad. Esta exterioridad caracteriza la alteridad, como figuración o expresión de una presencia plena, impactante, abierta sobre mí, como una bóveda, "aplastante" según la expresión levinasiana antes citada, de la cual difícilmente podríamos sustraernos. Lo interesante de esta caracterización es que ella es fuente de un asombro, iniciático, podríamos decir, en el que la subjetividad se abre a esta exterioridad del otro, se inicia a esta alteridad deslumbrante, al tiempo que se descubre en una relación inscrita en la huella de una especie de trauma. 
Demos un paso más. Despojada de su aserción espacial, la categoría de "exterioridad" que sostiene al otro viene a desentrañar una dimensión ética, a circunscribir éticamente la alteridad del otro. La conmoción que significa para la subjetividad que recibe a este otro es precisamente la marca de una afectación profunda, y que Lévinas determina como una desmesura ética (1990). Esta determinación ética de la exterioridad se impone como una indicación respecto de la vulnerabilidad y fragilidad extremas del otro, de su exclusión del mundo común, y que Lévinas tematiza en términos de la desnudez del otro, como una condición ontológica inicial, originaria, y en cuanto tal insuperable. Todo pasa como si el ser exterior fuese otra denominación para nombrar el sentido de la intemperie, de lo descampado como el sustrato emergente de la alteridad. Así lo sugiere Lévinas: "(...) desnudez del hombre, más exterior que el afuera del mundo (...) una desnudez que clama su carácter de extrañeza al mundo" (1993 266). En otras palabras, la exterioridad del otro sella la situación ontológica del expulsado del mundo, como forma de una indigencia sin superación, exiliado del mundo, o, como escribe Lévinas, "Expulsión fuera de la mundaneidad del mundo" (1975 54).

\section{Exterioridad y vulnerabilidad o la alteridad del hambriento}

No es difícil convenir que esta caracterización del otro en términos de una exterioridad se descubre estrechamente ligada a un lenguaje del desamparo y el abandono. Las tristes postales de los campos de concentración nazi, de los cuerpos de los prisioneros en los centros de tortura en el mundo entero, en nuestros países en Latinoamérica, en el desierto chileno, en donde fueron esparcidos los cuerpos de mujeres y hombres en la dictadura, todos ellos nos hablan de esa alteridad tan exterior que se pierde en los bordes de un mundo indiferente y afásico, que rima con el profundo espectáculo de la desolación.

La acepción ética de la categoría de "exterioridad" se profundiza en contenido y comprensión cuando, al circunscribir el ámbito de surgimiento del otro, éste se detiene en algunas figuraciones de la 
alteridad, extremadamente contemporáneas, y que Lévinas trabaja precisamente en función de esta comprensión del ser exterior, como un ser sin refugio, como pura exposición. Es ejemplar en este sentido cuando, bajo la pluma de Lévinas, el otro, en cuanto rostro, cristaliza en el pobre y el extranjero, pero también en el hambriento. Podríamos amplificar esta lista y nombrar hoy a los refugiados políticos, a los desplazados económicos, a los endeudados, a los excluidos del mundo entero.

Intentemos adentrarnos en el contenido del otro en cuanto el hambriento, categoría solidaria de la noción de "pobreza" como formas sustantivas de la alteridad del excluido. La figura del hambriento no es una figura entre otras, ella constituye un núcleo reflexivo determinante en la obra de Lévinas, en la medida en que teje el tenor moral y ético de esta filosofía. Lévinas no puede ser más claro cuando escribe que "reconocer al otro es reconocer un hambre" (1990 73), y es precisamente este reconocimiento la indicación de una dirección de sentido que indica la curvatura ética, en la que el afuera o el ser exterior comporta una eminencia de significación respecto de mi propio ser. Por otra parte, Lévinas es enfático en señalar que el pobre, como el hambriento, es aquel que "se retira del mundo" (1998 61). Esta condición de lo sin-mundo revela el carácter escandaloso de la pobreza; no se trata solamente -y como podría ser evidente- de que los hombres y mujeres en condición de pobreza y hambruna no dispongan de casa o de alimentos para la subsistencia. El asunto es tanto más grave porque han sido privados de mundo, es decir, de un horizonte desde donde poder inscribir un anhelo, proyectar un porvenir o despertar el horizonte de un mejor mundo posible. Estrictamente hablando, la experiencia del hambre es la experiencia de una expulsión y privación de mundo. "El hambre, escribe Lévinas, consiste en una desesperación de esta privación, [consiste] en no poder consolarse de un mundo" (1991 27) En otras palabras, la experiencia del hambriento es la experiencia de una desolación por ausencia de mundo. Conviene recordar que "desolación" remite a la voz latina solor, que significa confortar, aliviar, consolar. Desolación significa así ausencia de consuelo, de solaz, y por ello se prolonga por extensión a las ideas de desierto, abandono, destrucción. Y, no obstante, la ausencia de consuelo no solo traduce, 
sin más, la ausencia de un otro que podría estar en posición de darme consuelo o reparo, así como cuando declaramos sentirnos desolados ante la partida del amado o la amada. La desolación del hambriento es el testimonio de una ausencia de mundo (Castro, Gutiérrez 2017), de una ruptura del vínculo ontológico primero, en el que se juega la categoría de "vulnerabilidad".

Detengámonos en la categoría de "vulnerabilidad". Una de las características que define al sujeto contemporáneo es, sin duda, su estatuto ontológico de la vulnerabilidad. ¿Cómo entender esta vulnerabilidad? ¿En qué consiste ella? La salida del enclave de la inmanencia moderna y su horizonte trascendental no solo permite re-trazar la existencia como una que se da en-el-mundo, que no puede darse sino en ese estar inmiscuido en el mundo; además, reconfigura la posición de la subjetividad ante la escena mundana. La subjetividad aparece como una categoría volcada a la exterioridad, porque es ahí donde encuentra su horizonte constitutivo. En su libro Fenomenología de la Percepción, Merleau-Ponty declaraba el cese de las funciones del sujeto trascendental y la apertura a una nueva configuración de la subjetividad, que se descubre vinculada o implicada estructuralmente con el horizonte del mundo, que aparece como "el medio natural y el campo de todos mis pensamientos y de todas mis percepciones explícitas" (MerleauPonty 2008 11). Esta apertura al mundo decreta, al mismo tiempo, el desmoronamiento de la subjetividad volcada sobre sí misma, tenida por garante de las evidencias y certezas de este mismo mundo. En efecto, escribirá Merleau-Ponty, la verdad no "habita" (...) al "hombre interior"; mejor aún, no hay hombre interior, el hombre está en el mundo, es en el mundo que se conoce" (Ibíd.). El consecuente desarrollo de la afirmación el hombre "está en el mundo", conduce a Merleau-Ponty, a postular que nuestra relación con el mundo se diseña a la manera de un "tejido" (Ibíd.), de una trama intrincada, o, según el Merleau-Ponty de Lo visible y lo invisible, a la manera de un entrelazamiento o un quiasmo (1997). Esta manera de estar tejido con el mundo traduce la impronta de la filosofía merleaupontiana, según la cual el mundo ya no es más el simple correlato de una dimensión subjetiva interior, en la que el ser es subordinado al pensar y que, bajo este respecto, el ser del mundo puede ser abordado como aquello 
que yo simplemente percibo o pienso. En adelante, el mundo es "lo que yo vivo", en cuya traza se descubre la dimensión corporal como aquello mismo que engendra el tejido con el mundo. No podremos aquí desarrollar en profundidad la cuestión del cuerpo, pero solo digamos que esta idea de "mundo vivido" (Merleau-Ponty 2008 9), movilizada por esta fenomenología, traduce nuestra actitud primera hacia el mundo, según la cual, antes que teórico, nuestro primer contacto con el mundo es de orden experiencial y práctico, en otras palabras, pre-teórico. Ello determina no solo una nueva configuración del sentido del mundo, sino además permite vislumbrar la idea de vínculo con este mismo mundo, como una categoría comprensiva y estructurante de nuestra existencia. En otras palabras, afirmar nuestra existencia en el mundo es, al mismo tiempo, declarar la autoridad ontológica del vínculo, como una matriz comprensiva de la propia existencia en el mundo. Por ello, "Ser cuerpo, es estar anudado a un cierto mundo" (Id. 184), conjurando así, en este énfasis del vínculo con el mundo, el carácter abstracto del mundo, es decir naturalizado, matematizado, desvinculado del sujeto de la experiencia. La idea de "vínculo", movilizada por la dimensión corporal, lo que Merleau-Ponty nombra la "estructura de implicación" (Id. 185), aparece así como una condición ontológica relevante para el despliegue y comprensión de nuestra existencia, al tiempo que nos dice algo respecto de la condición de sujeto que aquí se juega. En efecto, en la complicidad con el mundo se decanta al mismo tiempo una cierta dimensión de vulnerabilidad constitutiva para el sujeto, como el resultado natural de nuestra implicación mundana. Ser en el mundo es estar lanzados a ese mismo mundo, expuestos a él, y hacer de esa apertura mundana nuestra primera condición existencial. En esta misma línea, alguien como Lévinas insistirá en esta apertura a la exterioridad, a la manera de una condición de estar arrojado en el mundo, pero asumiendo el horizonte de guerra y posguerra como aquello que tensiona dramáticamente esta apertura al mundo; en adelante estar-arrojado se convierte en un estar abandonado, dejando al sujeto en una orfandad patética respecto de sí mismo y de los otros. Bajo este respecto, la apertura existencial en el mundo conlleva precisamente el agregado de la vulnerabilidad en este primer sentido, según el cual el sujeto se 
descubre direccionado o entrelazado con el mundo. La vulnerabilidad es aquí una indicación de sentido y dirección del despliegue de nuestro ser en el mundo. Esto quiere decir que el mundo constituye algo así como un campo que afecta estructuralmente la existencia subjetiva, pero la afección del mundo es una afección que no destruye al sujeto, sino más bien lo constituye. En este primer sentido, hay que entender la vulnerabilidad en estricta relación con la idea de "vínculo", de ahora en adelante constitutivo de la relación sujeto-mundo, y que opera como condición de posibilidad de la configuración misma del sentido. Dicho en otros términos, la salida del sujeto moderno es la salida de la "subjetividad invulnerable" (Id. 10) de la modernidad, por antonomasia inmune al mundo que ella misma intenta construir o constituir, y en la que de Descartes a Kant, afirma Merleau-Ponty, se promueve la "absoluta certeza del yo por el yo" (Id. 9). Conciencia desafectada, invulnerable, inmune, neutral, potente, interior, desencarnada, desligada, espectadora, desvinculada; en ello reside la autoridad del sujeto moderno. Revertir esta condición ha sido sin duda uno de los esfuerzos de la filosofía contemporánea. Por ello declarar la salida del hombre interior es, al mismo tiempo, declarar la vulnerabilidad del sujeto, como aquel que puede ser afectado por el mundo en el cual se descubre arrojado, y que puede vivirlo ahora como un mundo poblado de matices, como un mundo bello, aburrido, horroroso o encantador.

Pero esta vulnerabilidad vinculante es puesta a prueba en ciertas condiciones existenciales límites, en las que la subjetividad parece recorrer un camino inverso, en el que no solo se produce una retracción del mundo, sino más bien su desvinculación más profunda. Es precisamente sobre esta situación, sobre esta perversión de la vulnerabilidad, que la alteridad del excluido resulta ilustradora en extremo, como la desvinculación más profunda, como un abandono que nos interpela. Esta vulnerabilidad, como hemos visto, es la marca del sujeto contemporáneo que, volcado en el mundo, descubre que su situación existencial consiste en estar expuesto a lo otro, y que, en cuanto tal, siguiendo aquí la etimología de la palabra vulnerable (vulnerabilis), puede ser dañado. En esta línea, Adriana Cavarero, en sintonía con Judith Butler, trabaja la idea de "vulnerabilidad del inerme" (2009 43) para indicar una exposición subjetiva a sentido único, 
sin reciprocidad, como si la vulnerabilidad fuese solo el índice de un potencial de daño. Así, la desolación de la que hablamos más arriba, tributaria del hambriento, de su materialidad sin mundo, se condice con la interrupción de toda forma de relación: con los otros, con las cosas, con las palabras, con el mundo y consigo mismo. Como si la vulnerabilidad -que es apertura al mundo- pudiese pervertirse en daño irreparable.

\section{Pensar a partir del excluido}

Volvamos a Lévinas. La dimensión práctica de esta metafísica levinasiana nos permite indagar en la eterna pregunta filosófica respecto del cómo se comienza a pensar y de las condiciones de emergencia de la filosofía. El gran mundo griego nos ha transmitido que el pensar propiamente filosófico pone en marcha un particular afecto. Los griegos nos han enseñado que el asombro es el afecto filosófico fundamental, pues es el signo inequívoco de un cambio de actitud, es el despertar de un comienzo y origen de una actitud propiamente filosófica; el asombrarse es el paso que deja atrás una dimensión irreflexiva -el sentido común, dirán algunos-, encaminándose a la actitud plenamente filosófica o racional. El asombro, como afecto filosófico, traduce así un cambio de mirada en el que lo que se tenía por dado, por incuestionado y certero, se presenta bajo una nueva perspectiva, y marca el acceso a una disposición de sentido hasta ahora apenas vislumbrada. Pero instalar el asombro como el afecto inaugural del pensar filosófico nada nos dice respecto de aquello que nos asombra. En efecto, la pregunta aquí será: ¿de qué nos asombramos? ¿Qué tipo de experiencias pueden impactar profundamente la subjetividad, al punto que en esta conmoción se despierte una disposición al saber, una apertura al pensamiento filosófico? En su texto, "Secularización y hambre" (1991) Lévinas retrotrae el asombro a una experiencia más bien escandalosa para la filosofía profesional, pues viene a contradecir las grandes tradiciones filosóficas. Pensemos por ejemplo en Descartes y sus Meditaciones metafísicas, en las que se nos enseña que un genuino ejercicio filosófico supone una vuelta a la subjetividad como punto de arranque para pensar el sentido, y que solo en esta 
vuelta reflexiva, en este movimiento que es una acción de ensimismamiento, podremos encontrar el hilo rojo que teje la sabiduría y que es fuente de amor para los filósofos, que es el camino que nos lleva a una auténtica reflexión filosófica, al tiempo que deja atrás las falsas evidencias y las certezas del sentido común. Este subjetivismo cartesiano es el prototipo de un pensamiento ensimismado, que hace de esta posición de recogimiento sobre sí la garantía y criterio para todo quehacer estrictamente filosófico. No olvidemos que el mismo Husserl, maestro indiscutido de Lévinas, ponderando este gesto cartesiano, comprende su filosofía como un neocartesianismo (Husserl 2016), hace de las meditaciones el prototipo de toda vía filosófica, dejando a la razón en la encrucijada de su más terrible soledad. En esta vía preguntamos: ¿de qué nos asombramos entonces? ¿De nuestra propia ignorancia, es decir de nosotros mismos? ¿Nos sorprendemos de un saber escondido en nosotros mismos, que, gracias a un acto de reflexión, de una vuelta a la subjetividad, hacemos madurar a través de un ejercicio autocentrado y nos sorprendemos o maravillamos de esta propia auto-comparecencia?

En otras palabras, ¿es el asombro un acto puramente teórico? A tal pregunta Lévinas responderá por la negativa, y pondrá en tela de juicio precisamente la apuesta de un origen del saber fundado en una estructura puramente teórica que menoscaba la trama de la vida práctica. Así, Lévinas inquiere críticamente: "¿El saber de occidente no es acaso la secularización de una idolatría?" (1991 21). Es decir, la secularización de un sentido filosófico que olvida la función de la alteridad, y el impacto que ella puede tener en la configuración del pensamiento racional. En efecto, para Lévinas no es del amor al saber que procede el saber, sino, sanciona el autor, procede "de las dificultades prácticas" (Id. 21). Se trata entonces de recoger, en la agitación de la vida en la tierra, los detonantes de un saber otro, pero sin hacer de ello una filosofía de mis dificultades y precariedades, una filosofía centrada en mi ser y en mi cuidado, en la que puede decantarse la indiferencia como un afecto constituyente de nuestro ser. La primera dificultad práctica es justamente el hambre del otro. En este sentido, pensar es asombrarse de la desolación del otro. Esto marca sin duda un cambio de paradigma que supone dejar atrás la "serenidad espe- 
culativa" (Lévinas 1991 23), propia de las filosofías centradas en las aventuras del Yo, para dar paso a esta filosofía fundada en lo que se puede llamar una "inquietud especulativa", que es la turbación que engendra el otro. Socialmente situado, hemos dicho que el hambriento es la forma de una desvinculación mundana, y por ello el hambre del hambriento es el testimonio encarnado de una ausencia de mundo. Esta privación es el fermento de toda acción especulativa.

El carácter indigente del otro está en la base de toda función de alteridad, y esta indigencia no solo es determinante de los contornos éticos del pensamiento de Lévinas, sino además dispone de efectos políticos que no podemos desconocer. Pues la pobreza, la miseria y el hambre son dimensiones condicionadas por otros, y, por ello, esta forma de alteridad situada se hunde de entrada en el terreno de la injusticia.

La alteridad del hambriento y del pobre prescribe la materialidad de los cuerpos sin mundo, $y$, en cuanto tal, se transforma en una demanda de justicia. Todo ello supone que lo que llamamos "mundo" nunca es una dimensión neutra o abstracta. Percibir un mundo, estar en el mundo, es ya encontrarse enredado en él, en una relación de inherencia con él y, en cuanto tal, encontrarse en una relación tonalmente tejida con él. Es descifrar ese mundo tal como se da para mí, descubriendo allí mi propia vida personal que es siempre colectiva, entramada con otros. Por ello, si junto con una ética, la política del otro hambriento fuese posible, no podría tratarse de una política de la limosna, de la súplica o la caridad, sino de una política de la demanda y emplazamiento al yo y a mi mundo por parte del otro. Es un reclamo de mundo. De esta manera, la justicia ética vira implacablemente en una demanda política, en una interdicción de olvido de esos cuerpos sin mundo, y el desafío es afinar el oído a la demanda del otro, y eso significa, como escribe Lévinas: "Escuchar su miseria que pide justicia (...)" (1990 237), y devolverle el mundo al otro, cumpliendo así la intuición levinasiana, según la cual ocuparse del hambre del otro es la "función primera de lo político" (Lévinas 1982 34) Por ello, prestar atención a la materialidad del otro es la indicación de una dirección ética que aparece bajo la forma de una interdicción de olvido del excluido, y que es a su vez una orientación de alteridad que funciona como un 
contrapeso necesario para comprender y renovar las configuraciones de lo político, lo social y sus formas institucionales.

Al final de este recorrido podemos afirmar, preliminarmente, que la materialidad de los cuerpos sin mundo profundiza su sentido en una semántica de la exclusión, que es el otro nombre de una vulnerabilidad que no reconoce en su traza la huella del mundo, sino más bien resuenan en ella los vestigios de un mundo roto, socavado de horizontes y expectativas. La alteridad del excluido no puede sino perturbar, incomodar, porque ella es la situación ética y política de un reclamo de mundo, que surge de la boca de un otro que se presenta como una indigencia, una extrema vulnerabilidad. Responder a ese reclamo que perturba es la fuente de la responsabilidad; responder a la miseria del otro es "ser responsable" (Lévinas 1990 237); en eso consiste la responsabilidad, en reconocer en el otro una vulnerabilidad hiperbólica, y en comprender, en consecuencia, que puedo dañar al otro. Por ello, la fragilidad aplastante del otro, que perturba mi ser, puede ser pensada como la condición de posibilidad y fuente de un saber otro, desplazado de mi propio yo, pero asentado en el otro. En esto consiste la materialidad de los cuerpos sin mundo, en resituar la función ética del hambre del otro, y en ver en esa materialidad indigente la fuente, quizás, de un nuevo humanismo, el del otro, el "humanismo de una humanidad hambrienta" (Lévinas 1991 24).

\section{Bibliografía}

Castro-Serrano, Borja y Gutiérrez Olivares, Claudia. "Intervención social y alteridad:una aproximación filosófica desde Lévinas", Andamios 14/33, enero-abril (2017): 217-239.

Chalier, Catherine y Abensour, Miguel. Emmanuel Lévinas. Paris: L'Herne, 1991.

Cavarero, Adriana y Agra S., Salvador. Horrorismo: Nombrando La Violencia Contemporánea. Rubí, Barcelona: Anthropos Editorial, 2009.

Gutiérrez Olivares, Claudia. "La Filosofía sin mundo de Emmanuel Lévinas", Revista de Filosofía Universidad de Chile LXVIII (2012): 103-114. 
Husserl, Edmund. Meditaciones cartesianas, trad. Mario Presas. Madrid: Técnos, 2016.

Lévinas, Emmanuel. Autrement qu'être ou au-delà de l'essence. Paris: Poche Essais, 1999.

Lévinas, Emmanuel. En découvrant l'existence avec Husserl et Heidegger. Paris: Librairie Philosophique J. Vrin, 2001.

Lévinas, Emmanuel. Totalité et infini. Essai sur l'extériorité. Paris: Poche Essais, 1990.

Lévinas, Emmanuel. Noms propres. Paris: Fata Morgana, 1975.

Lévinas, Emmanuel. Entre Nosotros. Ensayos para pensar en otro. Valencia: Pre-textos, 1993.

Lévinas, Emmanuel. De l'existence à l'existant. Paris: Librairie Philosophique J. Vrin, 1998.

Lévinas, Emmanuel. "Sécularisation et faim". Cahier de L'Herne. Emmanuel Lévinas, dir. Chalier, Catherine y Abensour, Miguel. Paris: L`Herne, 1991. 19-28.

Lévinas, Emmanuel. L'au-delà du verset. Lectures et discours talmudiques. Paris: Les Éditions de Minuit, 1982.

Merleau-Ponty, Maurice. Phénoménologie de la Perception. Paris: Gallimard, 2008.

Merleau-Ponty, Maurice. Le visible et I'nvisible. Paris: Gallimard, 1979.

Sartre, Jean-Paul. L'être Et Le Néant: Essai D'ontologie Phénoménologique. Paris: Gallimard, 1994. 